\title{
Actividad extraescolar para aprender a aprender: la robótica como herramienta educativa
}

\section{Extracurricular activity to learn to learn: robotics as an educational tool}

\author{
Florencio Castro, Juan Briegas, Sergio Ballester; Dolores González \\ Facultad Educación (UNEX)
}

\begin{abstract}
Resumen
Las actividades extraescolares tienen como objetivos educar y contribuir al desarrollo integral del alumno, estimular y potenciar sus aptitudes a través de la creatividad, la autonomía personal, las relaciones entre alumnos, el trabajo en equipo, la cooperación, etc. ¿Pero cómo se puede llevar esto acabo? En este artículo se describe la experiencia de la actividad robótica utilizada como herramienta educativa de aprendizaje, de desarrollo de capacidades y competencias. Entre los participantes se incluyen dos participantes con síndrome de Asperger (SA), un participante con Trastorno por Déficit de Atención e Hiperactividad (TDAH) y uno con trastorno del espectro autista (TEA).

Palabras clave: robótica, aprendizaje, inclusión, actividad, extraescolar
\end{abstract}

\begin{abstract}
Extracurricular activities aim to educate and contribute to the student's integral development, stimulate and enhance their skills through creativity, personal autonomy, student relationships, teamwork, cooperation, etc. But how can this be done? This article describes the experience of robotic activity used as an educational tool for learning, skills development and skills. Participants included two participants with Asperger's Syndrome (SA), one participant with Attention Deficit Hyperactivity Disorder (ADHD) and one with autism spectrum disorder (ASD).

Keywords: robotics, learning, inclusion, activity, out-of-school.Introducción
\end{abstract}

Uno de los objetivos principales de la educación es formar a niños desde las etapas más tempranas hasta la adolescencia, a lo largo de un recorrido educativo de aprendizaje que les guíe en la adquisición del conocimiento y la autonomía personal hasta alcanzar los inicios de la responsabilidad que le introduzcan paulatinamente en la edad adulta. Para ello no basta con disponer de conocimientos, éstos deben de ir acompañados de capacidades, habilidades, motivación y actitudes que les hagan personas completas capaces de desenvolverse en la sociedad y en el mundo futuro que les espera.

Aprender se define como la capacidad de cambiar la conducta como fruto de la experiencia, permitiendo al individuo adaptarse a nuevas situaciones ambientales y sociales (Gruar t i Massó, 2008).

La motivación, sin embargo, nos dirige hacia la el objetivo o la meta de una actividad, instigándola y manteniéndola (Pintrich y Schunk, 2006), impulsando al individuo a no rendirse y a luchar hasta conseguir el objetivo propuesto. La actitud, es la manifiesta disposición del ánimo que se expresa exteriormente. Es por ello que la motivación y la actitud para el aprendizaje, es decir, adecuación a los cambios en función de la tarea, son claves de cara a convertir a niños y niñas en personas competentes en un futuro. Las competencias integran de forma implícita elementos contextuales referidos al momento de aplicar los saberes a las tareas que la persona debe desempeñar (Zabala, 2007), y éste es el objetivo de la robótica educativa.

Desde la robótica educativa se establece, a partir de la teoría constructivista de Piaget, que no existe aprendizaje si no hay intervención del estudiante en la construcción del objeto de conocimiento (Ruíz, 2007).

Bajo esta idea, y aprovechando los grandes avances tecnológicos de las últimas décadas, números actividades se han ido desarrollando en el ámbito educativo orientadas a la implantación de esta nueva metodología de aprendizaje. Así, la robótica, como actividad extraescolar, no se establece como un fin, sino como un medio, como una herramienta que apoya el proceso de enseñanza-aprendizaje de forma lúdica, participativa y cooperativa entre los participantes de la actividad. De esta forma no solo trata de despertar vocaciones científico-técnicas, si no, al establecerse como medio de acción disponible del proceso educativo, por el carácter activo, participativo y cooperativo de los estudiantes busca desarrollar habilidades y competencias en los alumnos a través del juego (Barrera Lombana,N. 2014),

Es fundamental recalcar que el carácter lúdico de la actividad, en un ambiente desinhibido, cordial, amigable y ocioso favorece el aprendizaje. En este sentido, el juego funciona como una estrategia didáctica (Pizarro, 2007) de forma que los alumnos olvidan que están siendo observados y abandonan la timidez y las dificultades, haciendo que aprendan el contenido propuesto más fácilmente. Gracias al desarrollo de la "robótica educativa" y su aplicación como herramienta en el aprendizaje, el niño avanza en su didáctica como si de un "juego" se tratase, actuando como una actividad conductora de conocimientos y habilidades, que determina la evolución del niño (Vigotsky, 1988d:156).

En este artículo describimos los beneficios, capacidades y competencias obtenidos en el aprendizaje 
mediante la aplicación de la robótica como actividad extraescolar.

\section{Objetivos}

Describir los beneficios que proporciona la actividad extraescolar "robótica educativa" como herramienta de aprendizaje en niños con NEE.

\section{Método}

En este artículo se describen los beneficios obtenidos en la actividad extraescolar de robótica en un conjunto de alumnos en el que se integran cuatro alumnos con necesidades educativas especiales (NEE).

La metodología de aprendizaje llevada a cabo en esta actividad se adapta a edades, desde los 4 a los 16 años, y a través de la robótica y el trabajo colaborativo, complementan las necesidades curriculares de los jóvenes, abriéndoles nuevas oportunidades de completar su desarrollo.

La actividad basada en la metodología Lego ${ }^{\circledR}$ Education, en colaboración con el Massachusetts Institute of Technology, se desarrolla a lo largo del curso escolar mediante sesiones de 1 hora a 1,5 horas semanales, dependiendo de las edades.

Esta metodología seguida, diseñada por Lego $^{\circledR}$ Education en el año 1999, se basa en las 4 C's que forman parte de un proceso organizado para trabajar el proyecto educativo de Lego Education en las aulas:

Contemplar: se establece una conexión del conocimiento previo del alumno con el nuevo y se inserta una actividad práctica relacionada con el mundo real. También se atrae la atención del alumnado y se motiva como elemento imprescindible para un aprendizaje sólido.

Construir: Es el momento en el cual se registra una interacción entre mente y manos. El proceso de construcción proporciona un ambiente de aprendizaje fértil para el proceso de mediación por parte del facilitador que interviene en los conflictos y escucha diferentes ideas y opiniones de los grupos.

Conectar: Etapa en la que los participantes piensan en cómo funcionan las cosas, experimentando, observando, analizando y corrigiendo posibles errores, lo cual valida el proyecto y su viabilidad de aplicación en el mundo real. Aprender de los errores está en la esencia de la innovación.

Continuar: basada en el deseo natural del ser humano de aprender y conocer cosas. Se propone un nuevo desafío, fuertemente relacionado con el tema propuesto, que reta a los alumnos y los estimula a entrar en una espiral de aprendizaje donde en cada nivel superior se valoran los conocimientos previos, lo cual equilibra la relación de las habilidades con los desafíos.

Por tanto la metodología se basa en la superación de retos diarios, se establecen retos programados que los alumnos deben superar y que están orientados a desarrollar de forma práctica y didáctica sus habilidades motoras y cognitivas.

Dentro de las competencias trabajadas se encuentra la del trabajo en grupo y es por ello que la actividad se desarrolla en grupos generalmente de tres alumnos, quienes deben organizar internamente el grupo, asignarse sus propios roles de trabajo y cambiarlos cada cierto tiempo; a la vez deben discutir sus proyectos y llevarlos a cabo, consiguiendo como resultado final un buen clima de trabajo en el que prolifera el respeto por los compañeros y por sus opiniones.

A su vez esta metodología se desarrolla en un entorno lúdico, el alumno juega a la vez que aprende, "aprender jugando", siguiendo el valioso método pedagógico en el que el juego siempre es y será de interés para los participantes de cualquier edad

La investigación realizada se ha desarrollado con niños de diferentes colegios y de diferentes edades que han desarrollado sus actividades extraescolares en el mismo colegio donde cada uno desarrolla su etapa escolar. La metodología de la investigación ha sido de tipo descriptivo relacional de carácter cualitativo. El estudio se ha llevado a cabo mediante la técnica de la observación de los participantes por parte de los profesores o facilitadores y de la cuarta autora, coordinadora educativa y el análisis de cuestionarios por parte del grupo de investigación.

\section{Participantes}

Los participantes fueron 48 alumnos con diversas motivaciones distribuidos en distintos grupos de edades comprendidas entre los 4 y los 16 años. Dentro de la población se encontraban 2 alumnos con Trastornos de Déficit de Atención e Hiperactividad (TDAH), 1 alumno con síndrome de Asperger (AS), 1 alumno con Trastorno del espectro Autista (TEA).

\section{Materiales}

Los materiales utilizados para el desarrollo de las diferentes actividades fueron diversos materiales didácticos correspondientes al Set de Construcción de la casa que Lego® que permitieron a los alumnos construir y programar modelos simples LEGO ${ }^{\circledR}$ que se conectaban al ordenador, incluyendo multitud de piezas, sensores, etc., con diferentes actividades en diversas temáticas. Entre ellos se manejaron como material: $\operatorname{Lego}^{\circledR}$ Education Wedo 1.0, Lego ${ }^{\circledR}$ Education Wedo 2.0, Lego ${ }^{\circledR}$ Education Mindstorms, Lego ${ }^{\circledR}$ Education Build to Express, Scratch, Robot Bee-Bot, Lego Education Simple Machines, Lego ${ }^{\circledR}$ Education Early Machines, Lego ${ }^{\circledR}$ Education Learn to Learn, Lego ${ }^{\circledR}$ Education More to Math, Lego ${ }^{\circledR}$ Education Story Starter, Lego ${ }^{\circledR}$ Education Mecanismos Motorizados, Energías Renovables y Neumáticas.

\section{Instrumentos de recogida de datos}

Para evaluar los beneficios de la "robótica educativa" se emplearon diferentes cuestionarios (Cuestionarios de criterios diagnósticos del D.S.M. -IV y de la O.M.S (c.i.e.-10), cuestionarios de conducta de CONNERS para PROFESORES; (C.C.E.; Teacher`s Questionnaire, C. Keith Conners), cuestionario de conducta de CONNERS para Padres (C.C.I.; Parent's Questionnaire, C. Keith Conners), y fichas de valoración de la conducta de R.A.Barkley (1997)) cumplimentados por un profesor o facilitador de la actividad y por padres o tutores de los alumnos. Los datos se recogieron al comienzo de la 
actividad en el mes de octubre y al final del curso lectivo, tras nueve meses de actividad.

\section{Evaluación de la experiencia}

La valoración de la actividad se ha realizado de forma cualitativa a partir de la opinión de los facilitadores y de los padres.

Algunos de los alumnos que han desarrollado la actividad durante el curso 2016/2017 ya habían desarrollado la actividad anteriormente lo que conllevó una mayor predisposición al desarrollo del aprendizaje.

En lo que se refiere a los alumnos sin ninguna NEE los profesores o facilitadores observaron que alumnos que ya se conocían de otros cursos anteriores de la actividad extraescolar reforzaron su amistad a lo largo del curso de robótica, se complementaban y ayudaban para resolver con imaginación los retos que les planteaba (a menudo muy por encima del resto de los alumnos).

Uno de los alumnos que ya tenía destrezas adquiridas previamente en construcciones de Lego ${ }^{\circledR}$ al inicio del curso le sirvió para diferenciarse del resto de la clase rápidamente en el desarrollo de las actividades. Como curiosidad, esta diferenciación le llevo a un estado de "aburrimiento" con las construcciones más simples, por lo que el facilitador le sugirió el poder ayudar a los compañeros que estaban más rezagados, a lo que respondió con entusiasmo y, a menudo, aconsejaba y ayudaba a algún compañero durante las clases.

Otros alumnos se centraban al inicio del curso de la actividad en conseguir hacer los modelos que se les proponía paso a paso (no consintiendo bajo ningún concepto improvisar) pasando posteriormente, a lo largo del curso, a investigar por su cuenta y modificar los robots que se proponían en clase aplicando lo ya aprendido y con altas dosis de creatividad.

Otros al ir descubriendo el funcionamiento de algunos mecanismos simples desarrollaron su curiosidad más allá del simple juego, que era lo que les motivaba inicialmente.

Por otro lado, algunas de las capacidades que les ha costado más ha sido el trabajo en equipo, ya que todos querían construir ellos mismos y sin ayuda de otros compañeros, y aunque lo sobrellevan, todos pedían trabajo individual, desde el principio hasta el final del curso.

Las aptitudes que se han adquirido de forma paulatina durante el desarrollo de la actividad, han sido destrezas con las manos en la búsqueda de piezas, montaje y manipulación de los artefactos ya montados, capacidad de concentración, consiguiendo aislarse de todo lo que había alrededor.

Se dio el caso de que un niño de los más activos, muy impulsivo, desobediente, con un comportamiento desordenado, y a veces escandaloso, cuando se desarrollaba trabajos que realmente le agradaban, se concentraba totalmente en lo que estaba haciendo, sin levantar siquiera la mirada hacia sus compañeros que estuvieran armando jaleo, y realizando un trabajo perfecto en todos los casos, pidiendo ayuda al facilitador cuando lo necesitaba y finalizando la actividad que se hiciera en cada caso, tanto trabajando en equipo, como de forma individual, si bien de forma individual, la mayoría de las veces trabajaba de esta forma óptima.

En la faceta creativa, en términos generales, todos rebosaban creatividad. Con la actividad, en las ocasiones en las que les dejaba hacer creaciones propias de forma libre, los niños daban rienda suelta a su imaginación, poniendo mucha atención a lo que estaban haciendo así como un gran ímpetu en conseguir construir aquello que habían ideado y tratando de explicar posteriormente el trabajo que habían realizado.

Conseguir el logro del reto impuesto, ayudar a los compañeros en el montaje, consigue aportar a los alumnos seguridad en sí mismos, aumentando su autoestima.

En lo que respecta a los padres en niños sin NEE, ponen de manifiesto en términos generales que la participación en la actividad extraescolar ha suscitado en sus hijos un mayor interés por conocer el funcionamiento de las cosas, consiguen realizar más preguntas, y logran socializarse más y concentrarse en sus tareas diarias.

En los alumnos con TDAH se observó tanto por parte de los padres como por los facilitadores la mejoría de la capacidad de atención en una misma tarea, logrando aumentar el tiempo de concentración. Al principio de la actividad, estos alumnos, alternaban momentos de trabajo con otros de dispersión, observando que a medida que sucesión las sesiones, estos momentos de dispersión disminuían progresivamente hasta el punto de desaparecer.

Se observó un cambio en la aceptación y seguimiento de las normas en clase, así como en casa, dato que fue referido por las familias.

Inicialmente estos alumnos no admitían cambios de grupo y rol dentro del grupo, como obliga la actividad, sin embargo con el paso del tiempo estos alumnos se adaptaron a la norma que impone el cambio de grupo y de rol, sin ningún problema, aceptándola con agrado y realizando bien su trabajo.

Mejoró la relación social con los compañeros y con los amigos fuera del grupo, así como el comportamiento con ellos.

Los participantes diagnosticados con SA evolucionaron desde las primeras sesiones de la actividad, en las que mostraban impulsos incontrolados, no respetaban las normas, rechazaban a algunos compañeros como consecuencia de la falta de habilidades sociales, y presentaban baja tolerancia a la frustración, a un comportamiento reglado en el aula bajo las normas establecidas, la aceptación del error como base del aprendizaje y por tanto la tolerancia al fracaso y la frustración, así como el respeto hacia los propios compañeros .

De igual forma, el avance en el desarrollo de la actividad de robótica en el alumno TEA propició una mejora en sus relaciones con el resto de compañeros al aprender a controlar sus impulsos de desempeñar el rol que le apetecía en ese momento, y desapareciendo, casi en su totalidad, las estereotipias que tenía (ir al baño, abrir y cerrar puertas) las cuales sólo aparecían en momentos puntuales de nerviosismo o en el momento en el que se producía una situación distinta a la habitual; de igual forma se observó un aumento de su concentración 
tanto en casa como en el aula. En lo que respecta a la familia esta observó una mejora en la conducta en casa, mejora en la capacidad de atención en tareas de casa y en juegos con amigos y en la realización de tareas o actividades escolares, no percibiendo prácticamente mejoría en la canalización de los sentimientos de alegría o tristeza ni en la canalización del enfado o frustración

\section{Resultados}

Los resultados muestran que la actividad robótica educativa, como actividad extraescolar fomenta el interés por la Ciencia, la Tecnología, la Ingeniería, las Matemáticas, la documentación y la Programación informática en los participantes. Logra desarrollar habilidades y competencias en los alumnos como la creatividad, el razonamiento sistemático, la comunicación, la resolución de problemas, aumentando la autoestima de los participantes y mejorando la inclusión de los propios alumnos coincidiendo con lo indicado por Conchinha (2012).

A su vez la actividad extraescolar de robótica educativa ha permitido observar que ya desde la etapa infantil, los niños y niñas desarrollan habilidades para ordenar, clasificar o reconocer números, desarrollando además nociones de forma y espacio. Adquieren memoria visual y aprenden conceptos básicos sobre distintos tipos de máquinas simples. De igual forma, a través de la propuesta de solución de problemas y el seguimiento de divertidas fichas de actividades, se consigue involucrar a los participantes en procesos de cooperación, pensamiento creativo y comprensión lectora.

La exigencia de desarrollar las actividades en grupos de trabajo fomenta la explicación de ideas de resolución de los desafíos al resto de los compañeros favoreciendo el desarrollo lingüístico y potenciando la destreza de hablar en público.

El abordaje lúdico, ha permitido desarrollar el propio conocimiento de los participantes, y la concreción de desafíos animándolos a investigar, razonar, predecir y reflexionar, desarrollando su pensamiento crítico.

\section{Discusión}

En España, en el curso escolar 2015-2016 los alumnos con NEE en enseñanzas no universitarias que recibieron apoyo educativo superaron la cifra de 200.000.

Dentro de estos alumnos se incluyen los TDAH, trastorno de carácter neurobiológico originado en la infancia que implica un patrón de déficit de atención, hiperactividad y/o impulsividad y que en muchas ocasiones está asociado con otros trastornos comórbidos. Estos se presenta desde una edad temprana, generalmente antes de los 12 años, con una intensidad y frecuencia superior a la normal para la edad y en la etapa de desarrollo del niño, que deterioren o interfieran de forma significativa en el rendimiento del niño en dos o más de los ámbitos de su vida: escolar o laboral, familiar y social.

Por otro lado también se incluyen alumnos con el síndrome de Trastorno del Espectro Autista (TEA), este trastorno es una condición neurológica y de desarrollo que comienza en la niñez y dura toda la vida. Afecta cómo una persona se comporta, interactúa con otros, se comunica y aprende. Sus habilidades intelectuales, que pueden ir desde la discapacidad intelectual a capacidades intelectuales situadas en el rango medio, o superiores al mismo. Presentan alteraciones de las habilidades de comunicación verbal y no verbal, que pueden variar desde las personas que no emplean ningún lenguaje hasta las que tienen habilidades lingüísticas fluidas. A veces presentan comportamientos de forma repetitiva, $y$ pueden tener problemas para afrontar cambios en sus actividades y en su entorno. Estudios epidemiológicos realizados en Europa, apuntan que existe una prevalencia de aproximadamente 1 caso de TEA por cada 100 nacimientos (Autism-Europe aisbl 2015)

Igualmente dentro de alumnos NEE también se incluyen los síndrome de Asperger (SA), trastorno de origen neurobiológico enmarcado dentro de los Trastornos de Espectro del Autismo (TEA). Como tal, están presenten a lo largo del desarrollo de la persona, se pueden detectar desde edades tempranas y sus características se manifiestan a lo largo de toda la vida del individuo, con una importante variabilidad en función de los apoyos que reciba y sus propias estrategias de compensación adoptadas, entre otros factores . Tiene una incidencia de 3 a 7 de cada 1000 nacidos, especialmente en varones. El ratio mujer-hombre varía mucho dependiendo del estudio de referencia, desde 3-4:1 a 8:1. Presenta su principal área de alteración dentro de la relación social. La interacción con los demás no es ajustada, tanto por exceso (desinhibición), como por defecto (retraimiento y/o miedo al acercamiento), como por la forma de relacionarse con el entorno; su conducta no se adapta a los diferentes contextos y personas con los que se relaciona; posee una dificultad para inferir los sentimientos, emociones e intenciones de los demás, así como para expresar los propios. Es fuente en muchas ocasiones de conflictos por la incomprensión que genera ciertos comportamientos estereotipados, rutinas o dificultades de adaptación a los cambios.

Este tipo de alumnos recibe, una atención educativa diferente a la ordinaria debido a necesidades educativas especiales y/o a las dificultades específicas de aprendizaje que presenta.

Dentro de las actividades de apoyo, la robótica educativa se establece como una herramienta útil en el desarrollo de capacidades y habilidades con gran interés para el alumno en general y aplicación en sujetos o alumnos con NEE.

Una de las metodologías empleadas es la desarrollada en 1999 por Lego Education ${ }^{\circledR}$ que mediante la aplicación de las $4 \mathrm{c}^{\prime} \mathrm{s}$ permite un desarrollo secuencial de las competencias del alumno, Contemplando, al establecer una conexión del conocimiento previo del alumno con el nuevo, Construyendo al registrar una interacción entre mente y manos, Conectando al pensar en cómo funcionan las cosas, experimentando, observando, analizando y corrigiendo posibles errores, y Continuando, deseando aprender y conocer cosas, abordando nuevos desafíos, que los estimula en una espiral de aprendizaje. 
En nuestro estudio, en el que aplicamos la robótica como actividad extraescolar en 48 alumnos, nos centramos en pacientes con NEE, en los cuales la aportación de esta actividad mejora de forma notable sus capacidades, habilidades cognitivas, relacionales, sociales como así lo demuestra nuestros resultados.

Otros estudios (Conchinha, C; Honório Rodrigues, A.C.; Pires Nogueira; A; Correia de Freitas, J.;2016) reflejan que la robótica educativa "favorece el desarrollo de habilidades y competencias de los niños, así como la creatividad", y que la terapia basada en ella puede llegar a ser una "intervención interesante para el desarrollo de niños con TEA, niños con TDAH, niños con dificultades de aprendizaje específicas y niños con capacidades cognitivas superiores"

\section{Conclusión}

La robótica educativa es una herramienta útil y complementaria en los alumnos sin déficits cognitivos o conductuales y de gran complemento y ayuda en sujetos con NEE. Como actividad extraescolar la robótica educativa permite la integración de alumnos en el aula con distintas capacidades al propiciar el trabajo en equipo. Pensamos que esta fórmula de trabajo es adecuada para la inclusión de alumnos con NEE, al asumir que el error es fuente también del aprendizaje, y que no es motivo de frustración, y donde el profesor o facilitador, es un mero acompañante en el aprendizaje, siendo los participantes los verdaderos artífices de su propio avance en los conocimientos y habilidades.

Creemos que su aplicación, incluso en aulas de carácter ordinario, permitiría una mejor interrelación entre alumnos, un mejor desarrollo interpersonal, de conocimientos, de participación en equipo, incremento de autoestima y del respeto hacia sus compañeros y hacia las normas sociales establecidas.

\section{Referencias}

Barrera Lombana, N. (2014). Uso de la robótica educativa como estrategia didáctica en el aula. Praxis \& Saber - Vol. 6. Núm. 11 - Enero - Junio 2015 - Pág. 215-234

Boza Carreño, A.; Méndez Garrido, J.M. (2013). Aprendizaje Motivado en Alumnos Universitarios: Validacion y resultados generales de una escala. Revista de Investigación Educativa, 31 (2), 331-347.

Cortes, J. A., Arbelaez, O., y Mendoza, J. A. (2009). El entorno LEGO Mindstorms en la introducción a la robotica y la programacion. Scientia et Technica, 41(1), 42-45.

Gabriel Ocaña Rebollo. Robótica como asignatura en enseñanza secundaria. Resultados de una experiencia educativa.

Galvis, Á.H. (2007). Fundamentos de tecnología educativa. Costa Rica: Editorial EUNED.

Gura, M. (2011). Getting started with LEGO robotics. A guide for K-12 educators. Oregon: International Society for Technology in Education.

Gruart i Massó, A.(2008): ¿Por qué es el cerebro humano tan bueno para aprender y pensar? Córdoba, Biblioteca BenRosch, tomo 2 (El ser h u m a no).
Mena Rodríguez, F.E.; Salgado Muñoz, A.P.; Tamayo Marín, P.A. (2008). Estrategia Pedagógica basada en la lúdica y psicomotricidad aplicada en las áreas de castellano y ciencias naturales para centrar la atención de niños y niñas con TDAH de preescolar y básica primaria de la institución educativa ciudadela cuba.

Nelson Barrera Lombana, (2015). Uso de la robótica educativa como estrategia didáctica en el aula.

Ocaña, G. (2012). Robótica en el Aula. Almería: Consejería de Educación de la Junta de Andalucía.

Papert, S. (1980). Mindstorms: children, computers, and powerful Ideas. New York: Basic Books.

Pintrich, P.R. y Schunk, D.H. (2006). Motivación en contextos educativos. Madrid, Pearson.

Ricardo Baquero. (1997) Vigotsky y El Aprendizaje Escolar (1997). Grupo Editorial: Aique.

Rojas Vázquez, E; Poveda Peñuela, N.Y.; Hernández Ramírez, A.G. (2015). La lúdica de la palabra y el movimiento: estrategia que permite la construcción de aprendizaje holístico.

Ruiz-Velasco-Sánchez, E. (2007). Educa trónica: Innovación en el

aprendizaje de las ciencias y la tecnología. México: Ediciones Díaz Santos; UNAM.

Sanders, M. (2009). STEM, STEM Education, STEMmania. The Technology Teacher, 69(4), 20-26.

Santos, C. F. (2005). Um Estudo sobre Robótica Educacional usando Lego Mindstorm. (2005). 166p. Dissertação (Mestrado em Informática) - Universidade Federal do Espírito Santo, Vitória.

Vigotsky, L. S. (2003). Imaginación y creación en la edad infantil. Argentina: Editorial Nuestra América.

Zabala, A., Arnau, L (2007). Cómo aprender y enseñar competencias. 11 ideas clave. Barcelona, Editorial Graó, 2007 